\title{
Sonographic features of the lateral femoral cutaneous nerve in meralgia paresthetica
}

\author{
Xiaochen Shi, Feifei Liu, Fang Liu, Zheng Chen, Jiaan Zhu^ \\ Department of Ultrasound, Peking University People's Hospital, Beijing, China
}

Contributions: (I) Conception and design: J Zhu, X Shi; (II) Administrative support: None; (III) Provision of study materials or patients: X Shi, J Zhu, F Liu, Z Chen; (IV) Collection and assembly of data: X Shi, F Liu; (V) Data analysis and interpretation: F Liu, X Shi, J Zhu; (VI) Manuscript writing: All authors; (VII) Final approval of manuscript: All authors.

Correspondence to: Jiaan Zhu. Department of Ultrasound, Peking University People's Hospital, No. 11 Xizhimen South Street, Beijing 100044, China. Email: zhujiaan@pkuph.edu.cn.

Background: The diagnosis of meralgia paresthetica (MP) is usually based on clinical symptoms and physical examination. Therefore, the present study aimed to investigate the lateral femoral cutaneous nerve (LFCN) sonographic features in MP patients.

Methods: A total of 86 clinically suspected MP patients and 40 asymptomatic volunteers were prospectively recruited in the study. The sonographic features of the LFCN were observed by $18 \mathrm{MHz}$ high-frequency ultrasound. At the level of the anterior superior iliac spine, the cross-sectional area of the LFCN was measured.

Results: Of the 86 clinically suspected MP patients, 82 (95.3\%) had sonographic findings positive for MP. There were 54, 63, and 44 cases of abrupt caliber change, indistinct perineurium, and abnormal intraneural vascularity. The average value of the cross-sectional area of the LFCN at the level of the anterior superior iliac spine was $4.47 \pm 2.64 \mathrm{~mm}^{2}$, and the cut-off value was $2.65 \mathrm{~mm}^{2}$.

Conclusions: The sonographic diagnosis of MP may be achieved based on the following signs: nerve abrupt caliber change, indistinct perineurium of the nerve, intraneural vascularity, or increased crosssectional area.

Keywords: Meralgia paresthetica (MP); lateral femoral cutaneous nerve (LFCN); ultrasound

Submitted Feb 23, 2021. Accepted for publication Apr 25, 2021.

doi: 10.21037/qims-21-209

View this article at: http://dx.doi.org/10.21037/qims-21-209

\section{Introduction}

Meralgia paresthetica (MP) results from the compression of the lateral femoral cutaneous nerve (LFCN). The LFCN arises from the second and third lumbar plexus, travels downward lateral to the psoas muscle, and then crosses the iliacus muscle. MP occurs when the LFCN becomes compressed, and the symptoms are typically characterized by tingling, numbness, and burning pain of the lateral or the anterolateral aspect of the thigh (1).

The diagnosis of MP is usually based on the patient's symptoms and physical examination. Electrophysiological testing generally does not need to be used routinely (2). Imaging tests such as MRI, CT, or X-ray are not generally used to evaluate the LFCN directly. It is easier to identify the LFCN by ultrasound, and the anatomical variation of the LFCN can be viewed with high-frequency ultrasound

\footnotetext{
^ ORCID: 0000-0001-8700-639X.
} 
$(3,4)$. However, the clinical application of ultrasound in the LFCN mainly focuses on regional nerve block as a guide $(5,6)$. There are few reports regarding the ultrasound diagnosis of lateral femoral cutaneous neuritis. Powell et al. reported LFCN enlargement at the anterior superior iliac spine (ASIS) in patients with MP (7). A variety of factors can cause similar symptoms to MP. High-frequency ultrasound is widely used in peripheral neuropathy, which can clearly distinguish the internal structure of nerves. In order to better establish the basis of ultrasound evaluation of lateral femoral cutaneous neuritis, the purpose of our study was to identify sonographic abnormalities of the LFCN in cases of clinically suspected MP.

\section{Methods}

\section{Patient population}

A total of 86 clinically suspected MP patients and 40 asymptomatic volunteers were prospectively recruited in the study. The diagnosis of MP was based on patients' symptoms and physical examination, and a specialist evaluated all patients. Patients younger than 18 years old or with a previous history of hip surgery were excluded.

The control group consisted of 40 volunteers without any symptoms of pre-existing pain or dysesthesia in the distribution of the LFCN. Subjects with diabetes mellitus, pregnancy, peripheral neuritis, and a history of injury or surgery on the spinal column, pelvis, or groin were excluded from the study.

The study was conducted in accordance with the Declaration of Helsinki (as revised in 2013). The Ethical Committee approved this study of Peking University People's Hospital. All patients gave their written informed consent.

\section{Ultrasonography}

Each subject was positioned supine and was scanned by an investigator (18 years of neuromuscular ultrasound experience) using the Aplio i800 (Canon Medical Systems Corporation, Otawara, Tochigi, Japan), Siemens 3000 (Siemens Medical Solutions, Mountain View, USA), Aixplorer (Supersonic Imagine, France), and Mylab 90 (Esaote, Genoa, Italy) with an $18 \mathrm{MHz}$ linear array transducer. The ultrasound scan method of the LFCN was performed according to the previous report of our group (3), and the intermuscular space between the tensor fasciae latae muscle and the sartorius was used as an initial sonographic landmark. Then, sonographic features of the LFCN were observed, including abrupt caliber change at the ASIS, indistinct perineurium, and abnormal intraneural vascularity (color blood flow signal detected by ultrasound in the LFCN). At the level of the ASIS, the cross-sectional area (CSA) of the LFCN was measured. All the asymptomatic volunteers were scanned bilaterally. Evaluation of the sonographic images was performed by the 3 radiologists (JZ, $\mathrm{XS}$, and FL, with 3-18 years of experience in peripheral nerve ultrasound) with no knowledge of clinical findings. Each reader had experience with more than 30 cases of LFCN ultrasonography.

\section{Statistical analyses}

Quantitative data were expressed by the mean \pm standard deviation and qualitative data by percentages. The normally distributed continuous variables were compared using Student's $t$-test. The categorical variables were compared with the Chi-squared test. Inter-observer agreement was calculated by intraclass correlation coefficients, unweighted kappa statistics, and overall agreement. A kappa value of $0-0.20$ was considered poor; $0.21-0.40$, fair; $0.41-0.60$, moderate; 0.61-0.80, good; and 0.81-1.00, excellent agreement. No significant differences were observed between the right and the left sides concerning the CSA of the LFCN, and the parameters from the bilateral sides were combined for the final analysis. We calculated sensitivities, specificities, and positive and negative predictive values of each ultrasound index. A receiver operating characteristic (ROC) curve was generated to determine the optimal cutoff value for the CSA in differentiating MP patients from healthy controls. All statistics were performed using SPSS software (version 22.0). A value of $\mathrm{P}<0.05$ was considered statistically significant.

\section{Results}

There were no statistically significant differences regarding sex, age, and BMI between MP patients and controls. The demographic characteristics of participants are shown in Table 1.

Each nerve was identified satisfactorily using ultrasound in all participants. Of the 86 clinically suspected MP patients, $82(95.3 \%)$ had sonographic findings positive for MP. The LFCN had an abrupt caliber change at the ASIS in 54 cases (Figure 1). The indistinct perineurium of 
Table 1 Demographic characteristics of participants

\begin{tabular}{lccc}
\hline Variable & MP patients & Control group & P value \\
\hline Number of LFCNs & 86 & 80 (bilateral) & \\
Male/female & $56 / 40$ & $50 / 30$ & 0.16 \\
Age (years, mean $\pm \mathrm{SD})$ & $40.29 \pm 10.8$ & $37.6 \pm 11.9$ & 0.7 \\
$\mathrm{BMl}\left(\mathrm{kg} / \mathrm{m}^{2}\right)$ & $25.7 \pm 4.1$ & $24.2 \pm 4.9$ & 0.2 \\
\hline
\end{tabular}

MP, meralgia paresthetica; LFCN, lateral femoral cutaneous nerve; BMI, body mass index.

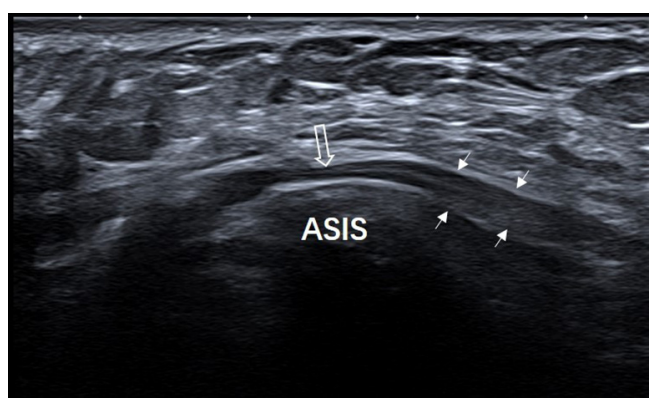

Figure 1 Longitudinal ultrasound image shows the abrupt caliber change of the nerve (empty arrow). Arrow, lateral femoral cutaneous nerve; ASIS, anterior superior iliac spine.

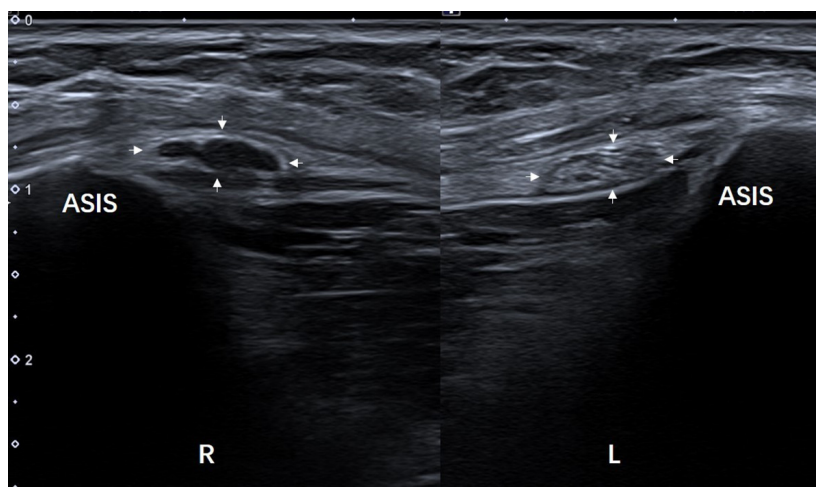

Figure 2 Transverse ultrasound image shows the indistinct perineurium of the right nerve (left shows a normal nerve). Arrow: lateral femoral cutaneous nerve. R: right; L: left; ASIS: anterior superior iliac spine.

the LFCN on the transverse sonogram made the nerves hypoechogenic, which was noted in 63 cases (Figure 2). Abnormal intraneural vascularity was noted in 44 cases (Figure 3). Also, 2 cases of schwannoma of the LFCN were found in the anterolateral part of the thigh near the groin (Figure 4). In the control group, intraneural vascularity was

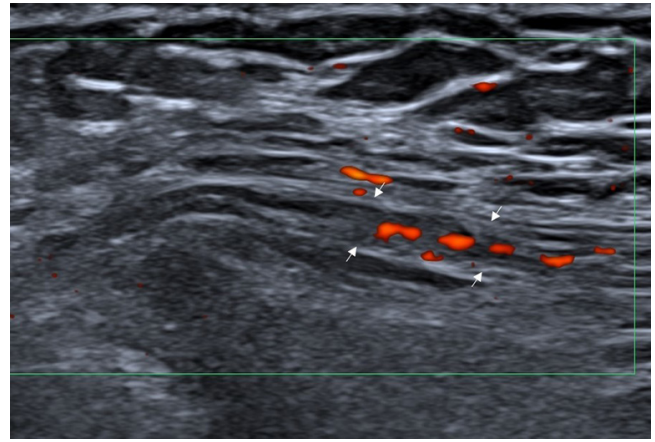

Figure 3 Longitudinal ultrasound image shows intraneural vascularity. Arrow: lateral femoral cutaneous nerve.

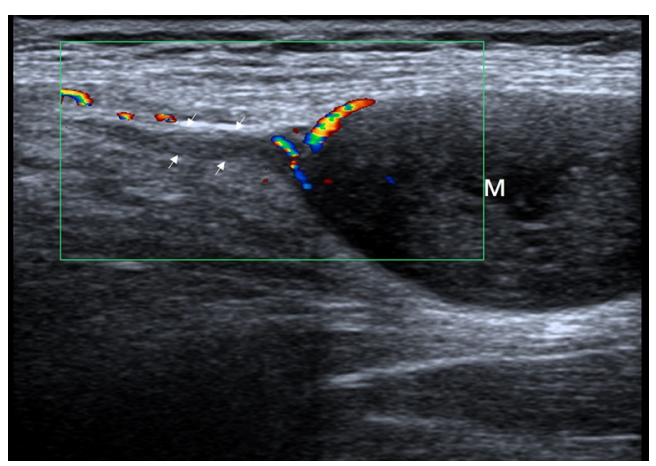

Figure 4 Longitudinal ultrasound image of the schwannoma. Arrow: lateral femoral cutaneous nerve.

found only in 6 cases, and there were no other abnormal sonographic findings. The CSA of the LFCN at the level of the ASIS was significantly larger in the MP group compared to the control group $(\mathrm{P}<0.0001)$, and the average values were $4.47 \pm 2.64$ and $1.70 \pm 0.63 \mathrm{~mm}^{2}$, respectively $(\mathrm{P}<0.0001)$. A ROC curve with a criterion of LFCN CSA of $>2.65 \mathrm{~mm}^{2}$ resulted in $73.3 \%$ sensitivity, $91.2 \%$ specificity, and an area under the curve of $0.909(0.868-0.950)$ (Figure 5). The sensitivity and specificity of different indexes in the diagnosis of MP are shown in Table 2.

The inter-observer variability correlation coefficients for CSA, abrupt caliber change, indistinct perineurium, and intraneural vascularity were $0.908,0.992,0.885$, and 1.0 , respectively.

A total of 52 patients underwent an ultrasound-guided nerve block of the LFCN (Figure 6), with all patients reporting symptom reduction or relief. Two cases of schwannoma were surgically removed. The others received medicine or physical therapy, and their symptoms were improved to 


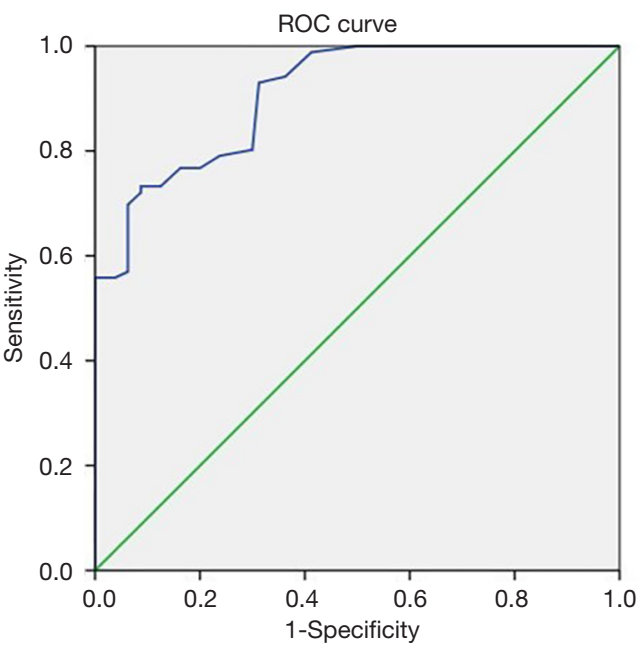

Figure 5 ROC curve of the CSA for determining patients with meralgia paresthetica. ROC, receiver operating characteristic; CSA, cross-sectional area.

Table 2 Sensitivity and specificity of different indexes in the diagnosis of MP

\begin{tabular}{lcccc}
\hline Index & Sensitivity (\%) & Specificity (\%) & PPV & NPV \\
\hline ACC & 62.79 & 100 & 100 & 71.43 \\
IP & 55.81 & 100 & 100 & 67.80 \\
V & 51.16 & 95.06 & 93.62 & 64.71 \\
CSA & 73.30 & 91.20 & 90 & 76.04 \\
\hline
\end{tabular}

MP, meralgia paresthetica; PPV, positive predictive value; NPV, negative predictive value; ACC, abrupt caliber change; IP, indistinct perineurium; $V$, intraneural vascularity; CSA, cross-sectional area.

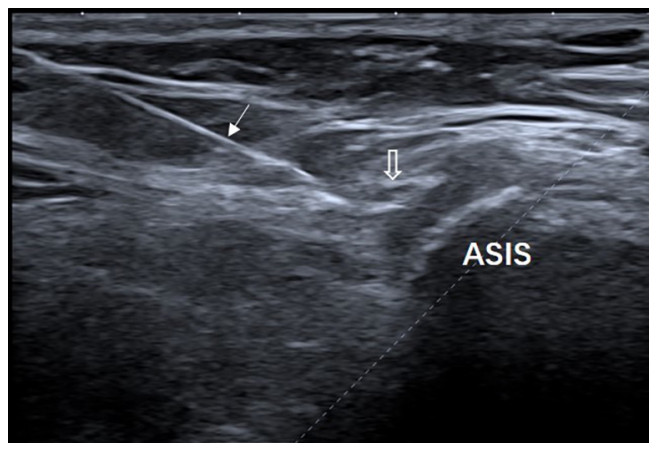

Figure 6 Ultrasound-guided nerve block. Arrow: needle; empty arrow: lateral femoral cutaneous nerve; ASIS, anterior superior iliac spine. varying degrees. Of the 86 clinically suspected MP patients, 4 had no positive sonographic findings. One patient had shingles 6 months ago, and 3 patients dropped out.

\section{Discussion}

MP is also called lateral cutaneous nerve neuralgia (8) and was previously known as Bernhard-Roth syndrome (9). MP appears to be more common than previously thought, and the incidence rate of MP is around 3.26 or 4.3 per 10,000 person-years (10). Although MP is a clinical diagnosis, noninvasive ultrasound examination can provide an important basis for imaging diagnosis. Our results demonstrate that if one of these abnormal sonographic features is present, that is, nerve abrupt caliber change, indistinct perineurium of the nerve, intraneural vascularity, or increased CSA, MP can be diagnosed by ultrasound.

Several studies on the ultrasonic diagnosis of MP; however, all these studies used CSA of the LFCN at the ASIS as the only index to evaluate MP. Powell et al. reported a CSA of $9 \mathrm{~mm}^{2}$ versus $3 \mathrm{~mm}^{2}(\mathrm{SD} \pm 2.31)$ in positive cases and normal controls, respectively (7). Suh et al. reported that the median value of the CSA of the LFCN in MP patients was $11 \mathrm{~mm}^{2}$ (11). The optimal cut-off value of the previous 2 studies for the diagnosis of MP was $5 \mathrm{~mm}^{2}$. Another researcher reported that the measured CSA value was $4 \mathrm{~mm}^{2}$ in MP patients (12). In our study, a cut-off value of $2.65 \mathrm{~mm}^{2}$ was considered to be pathological in MP patients. The reason for this difference may be related to the sample source and data size. More importantly, if CSA was used as the ultrasound parameter alone, there may be more false-negative results. Powell et al. found that $30 \%$ of suspected MP cases had no ultrasound findings suggestive of MP (7).

The abrupt caliber change of the LFCN is the direct sign of nerve compression, and ultrasound can usually find fibrous tissue around the nerve. With the advent of $18 \mathrm{MHz}$ high-frequency ultrasound, it is possible to evaluate nerve echotexture. The indistinct perineurium of the LFCN on the transverse sonogram made the nerves hypoechogenic and increased intraneural vascularity may further aid patients with neuropathy caused by LFCN compression, which was similar to the ultrasound findings of carpal tunnel syndrome (13).

Numerous factors may contribute to the mechanical damage of the LFCN, and ultrasound can find direct 
morphological signs at the ASIS. We found 2 schwannomas of the LFCN, which were in the anterolateral part of the thigh near the groin. There has been a previous report regarding lipoma overlying the sartorius muscle (14). We consider that the key to the ultrasonic evaluation of MP is the standard LFCN scan methodology. It is important to visualize the course of the LFCN, and not only at the level of the ASIS. We recommended dynamic scanning from the intermuscular space between the tensor fasciae latae muscle and the sartorius to the ASIS (3). In this way, it can not only find lesions, but it is also easier to find the relationship between lesions and nerves.

The most important limitation of this study was the lack of a gold standard for the diagnosis of MP. However, in most cases, the diagnosis of MP is primarily based on medical history and a physical exam. Electromyography and nerve conduction studies are not routinely recommended, and these tests might be performed to exclude other disorders when the diagnosis is not clear, or there are other causes for the symptoms. Another limitation was that we did not observe the relationship between the anatomical variation of the LFCN and MP. Although this study mainly emphasized changes in sonograms, anatomical variations are often encountered during ultrasound examination or clinical treatment $(15,16)$. Detecting anatomical variations of the LFCN by ultrasound helps identify the LFCN during surgery (15).

In summary, the sonographic diagnosis of MP may be achieved based on the following signs: abrupt caliber change, indistinct perineurium of the nerve, intraneural vascularity, or increased CSA.

\section{Acknowledgments}

Funding: This study was supported by funding from the National Natural Science Foundation of China (81701712, 82071930).

\section{Footnote}

Conflicts of Interest: All authors have completed the ICMJE uniform disclosure form (available at http://dx.doi. org/10.21037/qims-21-209). The authors have no conflicts of interest to declare.

Ethical Statement: The authors are accountable for all aspects of the work in ensuring that questions related to the accuracy or integrity of any part of the work are appropriately investigated and resolved. The study was conducted in accordance with the Declaration of Helsinki (as revised in 2013). This study was approved by the Ethical Committee of Peking University People's Hospital. All patients gave their written informed consent.

Open Access Statement: This is an Open Access article distributed in accordance with the Creative Commons Attribution-NonCommercial-NoDerivs 4.0 International License (CC BY-NC-ND 4.0), which permits the noncommercial replication and distribution of the article with the strict proviso that no changes or edits are made and the original work is properly cited (including links to both the formal publication through the relevant DOI and the license). See: https://creativecommons.org/licenses/by-nc-nd/4.0/.

\section{References}

1. Sanjaya A. Meralgia paresthetica. Postgrad Med 2020;132:1-6.

2. Seror P, Seror R. Meralgia paresthetica: clinical and electrophysiological diagnosis in 120 cases. Muscle Nerve 2006;33:650-4.

3. Zhu J, Zhao Y, Liu F, Huang Y, Shao J, Hu B. Ultrasound of the lateral femoral cutaneous nerve in asymptomatic adults. BMC Musculoskelet Disord 2012;13:227.

4. Damarey B, Demondion X, Boutry N, Kim HJ, Wavreille G, Cotten A. Sonographic assessment of the lateral femoral cutaneous nerve. J Clin Ultrasound 2009;37:89-95.

5. Nielsen TD, Moriggl B, Barckman J, Kølsen-Petersen JA, Søballe K, Børglum J, Bendtsen TF. The Lateral femoral cutaneous nerve: description of the sensory territory and a novel ultrasound-guided nerve block technique. Reg Anesth Pain Med 2018;43:357-66.

6. Finneran JJ, Swisher MW, Gabriel RA, Fiallo A, Hamilton JR, Lee JG, Ilfeld BM. Ultrasound-guided lateral femoral cutaneous nerve cryoneurolysis for analgesia in patients with burns. J Burn Care Res 2020;41:224-7.

7. Powell GM, Baffour FI, Erie AJ, Puffer RC, Spinner RJ, Glazebrook KN. Sonographic evaluation of the lateral femoral cutaneous nerve in meralgia paresthetica. Skeletal Radiol 2020;49:1135-40.

8. Dias Filho LC, Valença MM, Guimarães Filho FA, Medeiros RC, Silva RA, Morais MG, Valente FP, França SM. Lateral femoral cutaneous neuralgia: an anatomical insight. Clin Anat 2003;16:309-16.

9. Harney D, Patign J. Meralgia paresthetica: diagnosis and management strategies. Pain Med 2007;8:669-77. 
10. Parisi TJ, Mandrekar J, Dyck PJ, Klein C. Meralgia paresthetica: relation to obesity, advanced age and diabetes mellitus. Neurology 2011;77:1538-42.

11. Suh DH, Kim DH, Park JW, Park BK. Sonographic and electrophysiologic findings in patients with meralgia paresthetica. Clin Neurophysiol 2013;124:1460-4.

12. Aravindakannan T, Wilder-Smith EP. High-resolution ultrasonography in the assessment of meralgia paresthetica. Muscle Nerve 2012;45:434-5.

13. Mallouhi A, Pülzl P, Trieb T, Piza H, Bodner G. Predictors of carpal tunnel syndrome: accuracy of gray-scale and color Doppler sonography. AJR Am J Roentgenol

Cite this article as: Shi X, Liu F, Liu F, Chen Z, Zhu J. Sonographic features of the lateral femoral cutaneous nerve in meralgia paresthetica. Quant Imaging Med Surg 2021;11(10):4269-4274. doi: 10.21037/qims-21-209
2006;186:1240-5.

14. Rau CS, Hsieh C, Liu Y, Wang L, Cheng M. Meralgia paresthetica secondary to lipoma. J Neurosurg Spine 2010;12:103-5.

15. de Ruiter GCW, Wesstein M, Vlak MHM. Preoperative Ultrasound in patients with meralgia paresthetica to detect anatomical variations in the course of the lateral femoral cutaneous nerve. World Neurosurg 2021;149:e29-35.

16. Aszmann OC, Dellon ES, Dellon AL. Anatomical course of the lateral femoral cutaneous nerve and its susceptibility to compression and injury. Plast Reconstr Surg 1997;100:600-4. 